\title{
Levodopa improves time-based prospective memory in Parkinson's disease
}

\author{
ALBERTO COSTA,${ }^{1}$ ANTONELLA PEPPE,${ }^{1}$ LIVIA BRUSA,${ }^{2}$ CARLO CALTAGIRONE,,${ }^{1,3}$ \\ ILARIA GATTO ${ }^{1}$ AND GIOVANNI AUGUSTO CARLESIMO ${ }^{1,3}$ \\ ${ }^{1}$ Istituto di Ricovero e Cura a Carattere Scientifico Fondazione Santa Lucia, Roma, Italy \\ ${ }^{2}$ Ospedale Sant'Eugenio, Roma, Italy \\ ${ }^{3}$ Clinica Neurologica, Università di Roma "Tor Vergata", Roma, Italy \\ (Received October 3, 2007; Final Revision April 2, 2008; Accepted April 2, 2008)
}

\begin{abstract}
The present study was aimed at investigating the effect of acute levodopa administration on the performance of a group of individuals with Parkinson's disease (PD) on a time-based prospective memory task. Twenty PD patients and 15 healthy controls were administered a task that required executing three actions after $10 \mathrm{~min}$ had elapsed in three consecutive trials. Scores were computed for correct recall of the intention to perform the actions and for correct execution of the actions. PD participants were evaluated after a 12-hr drug wash-out in two conditions: (1) after levodopa administration ("on"); (2) without drug administration ("off"). In the "on" condition, PD patients were significantly more accurate in retrieving the intention to perform the actions than in the "off" condition and their performance was actually comparable to that of healthy controls. The increased accuracy in complying with the prospective memory task following levodopa medication supports the idea that dopamine depletion plays a role in the prospective memory deficits observed in PD patients. (JINS, 2008, 14, 601-610.)
\end{abstract}

Keywords: Cognitive processes, Prospective intention, Executive functions, Neurochemical alterations, Neurobiology, Frontal-striatal loops

\section{INTRODUCTION}

Prospective memory refers to the ability to execute intentions at a certain later time (i.e., time-based prospective memory) or when some external event occurs (i.e., eventbased prospective memory) (Einstein \& McDaniel, 1990). In the past few years, there has been growing interest in investigating prospective memory deficits in brain damaged individuals, because a failure to carry out previously planned intentions may have a great impact on every day life (Kliegel \& Martin, 2003). Indeed, a prospective memory deficit was reported as a primary determinant of loss of functional independence in patients with neurological diseases (Burgess \& Shallice, 1997; Burgess et al., 2000).

Prospective memory requires multiple abilities and is particularly demanding of those involving declarative memory

Correspondence and reprint requests to: Alberto Costa, Fondazione IRCCS S. Lucia, Via Ardeatina 306-00179, Roma, Italy. E-mail: a.costa@ hsantalucia.it and executive functions (Knight, 1998). According to Einstein and McDaniel (1990, 1996), two main components are involved in performing a prospective memory task. The first component involves recalling the intention to do "something" at the appropriate moment. It also requires the continuous recruitment of attentional resources to shift from an ongoing activity to the execution of the delayed intention when the required amount of time has elapsed or the cueing event has occurred. The second component requires the ability to store and retrieve the detailed contents of the encoded intention (the actual plan of action) once the intention has been activated. Attentional resources and executive abilities are mainly involved in retrieving the intention while the recollection of the specific actions to be performed would be mediated to a greater extent by the same declarative memory system that is responsible for the retrieval of all facts or events (Einstein \& McDaniel, 1996; Knight, 1998). Neuropsychological evidence in keeping with this view has been provided. Indeed, individuals with a dysexecutive syndrome due to frontal lobe damage are particularly poor in 
initiating the programmed actions (Burgess, 2000; Burgess $\&$ Shallice, 1997). On the other hand, in a group of subjects with severe closed-head injury who showed an impairment of both the intention retrieval and the recollection of the specific actions to be performed, a significant association was found between performance on episodic memory tasks and the ability to remember the specific details of the plan in a prospective memory task (Carlesimo et al., 2004). Functional neuroimaging investigations have suggested a crucial role of polar and dorsolateral prefrontal regions in the genesis and retention of prospective intentions (Burgess et al., 2001, 2003).

Recent reports have consistently documented a deficit in prospective memory function in patients with Parkinson's disease (PD). Impaired retrieval of the prospective intention in nondemented PD patients has been documented both on event-based (Katai et al., 2003; Kliegel et al., 2005) and time-based (Costa et al., in press) tasks, and there is evidence of an association between poor prospective memory and executive dysfunction in PD (Costa et al., in press). In $\mathrm{PD}$, cognitive impairment is primarily mediated by cortical pathology (Bohnen et al., 2006; Emre, 2003; Gibb et al., 1989). However, the evidence that treatment with L-dopa improves performance on some mental tasks (e.g., Costa et al., 2003; Lewis et al., 2005) suggests that some facets of cognitive dysfunction in PD are actually related to dopamine depletion (Owen, 2004). In the case of prospective memory, the hypothesis can be advanced that the involved frontal networks are rendered dysfunctional either by dopamine depletion in the target cortical structures or by dysregulation of frontal-striatal networks as a result of dopamine depletion in subcortical structures. Cortical depletion of dopamine in prefrontal areas due to the degeneration of the mesocortical and mesolimbic systems has been reported (see Owen, 2004; Cools et al., 2002). Although early dopamine depletion in the putamen may account for the motor deficits associated with PD, it is commonly held that dopamine deficiency in the caudate nucleus could be implicated in the cognitive dysfunction (Kish et al., 1988). In the caudate nucleus the depletion of dopamine is greatest in the rostrodorsal extent of the head, a region strongly connected to the dorsolateral prefrontal cortex, and only late in the disease course does depletion occur in the more ventral part that is preferentially connected to ventral prefrontal cortex (Agid et al., 1993; Yeterian \& Pandia, 1991). The presence of cognitive deficits generally associated with dorsolateral prefrontal dysfunction (e.g., executive and working memory impairments) is a frequent finding even in the early stages of PD (Costa et al., 2003; Owen, 2004) and could therefore plausibly be related to the pattern of striatal dopamine depletion characteristic of PD.

Given previous evidence that dopamine depletion in the brains of patients with PD leads not only to motor symptoms but also to some cognitive deficits, the present study sought to determine whether the acute administration of levodopa (L-dopa) would improve the performance of these patients on a time-based prospective memory task. In this study, a group of PD patients performed a time-based prospective memory task twice: once following a period of L-dopa therapy withdrawal; and once immediately after the oral administration of $200 \mathrm{mg}$ of L-dopa. The experimental task [which in a previous study proved to be particularly sensitive in detecting time-based prospective memory deficits in a PD population (Costa et al., in press)] was specifically designed to assess the ability to recall the intention separately from the capacity to retrieve the specific actions to be performed. Based on the hypothesis of an association between dopamine depletion and prospective memory dysfunction in PD, we predicted that the PD patients' performances would be better in the on-state, after taking L-dopa, than in the off-state. Moreover, in view of the especially critical role played by frontal circuits in keeping active the intention to perform the planned action (Burgess et al., 2001), the L-dopa related performance improvement was expected to be particularly evident in the accuracy of the intention retrieval.

\section{MATERIAL AND METHODS}

\section{Participants}

Twenty individuals affected by idiopathic PD and 15 control subjects (CS) of comparable age and education participated in the study. All participants gave their informed consent and the study was approved by the local Ethics Committee. The diagnosis of idiopathic PD was made by an expert neurologist based on (1) the presence of at least two of the four cardinal symptoms; (2) good chronic response to L-dopa treatment (Hughes et al., 1992). Exclusion criteria for the PD group included (1) Hoehn and Yahr's (1967) score $>2.5$; (2) presence of severe systemic or metabolic diseases; (3) evidence of large vessel distribution cerebral infarcts on CT and/or MRI scans; (4) a Major Depressive Disorder based on the criteria of the Diagnostic and Statistical Manual of Mental Disorders, fourth edition (DSM IV) (American Psychiatric Association, 2000); (5) a history of other neurological disorders, head trauma, or substance abuse; (6) severe functional impairment of the autonomic nervous system; (7) use of other active central nervous system drugs such as stimulants, sedatives, antidepressants, antipsychotics, or anticonvulsants; (8) dementia based on clinical examination according to DSM-IV criteria (American Psychiatric Association, 2000) and a Mini-Mental State Examination (MMSE) score $\leq 24$ (Folstein et al., 1975). While performing the experimental task, participants were also given some tests of executive functions, attention and short-term memory (see below). Analysis of PD patients' performances on these tests revealed that two patients scored more than 1.5 SDs below the healthy controls' mean on the interference condition of the Stroop test (Stroop, 1935) and one patient scored below normal on the Trail Making Test part B (Reitan, 1958). However, as these patients had an MMSE score $>28$, indicating the absence of dementia, they were not excluded from the study. All PD subjects were 
taking L-dopa combined with a dopamine agonist: ropinirole, pramipexole, or cabergoline (means, 9, 2.1, and $4 \mathrm{mg}$, respectively). The mean daily dosage of the levodopa treatment was $250 \pm 125 \mathrm{mg}$. Exclusion criteria for the CS group included: (1) suspected dementia based on clinical examination or a Mini Mental State Examination score $\leq 24$; (2) taking medication with central nervous system effects such as stimulants, sedatives, antidepressants, antipsychotics or anticonvulsants; (3) a history of head trauma, substance abuse, psychiatric or neurological illness. Clinical and demographic characteristics of the PD and CS groups are reported in Table 1 .

\section{General design}

The experimental procedure and inter-current tasks (see below for a description) were administered to PD subjects in two different treatment conditions performed on different days and separated by an interval of at least two weeks. In the "off" therapy condition, the PD participants were evaluated in the morning after overnight therapy withdrawal, that is, at least $12 \mathrm{hr}$ after last medication dose, and the prospective memory task was performed without any prior drug administration. In the "on" therapy condition, the prospective memory task was administered to PD subjects 20-30 min after oral administration of $200 \mathrm{mg}$ of L-dopa-corresponding to 1.5 times the average morning dose, combined with $50 \mathrm{mg}$ of an inhibitor of L-dopa metabolism and following pretreatment with domperidone to prevent nausea (40 mg p.o. on the day before the test, and 30 mg p.o. $15 \mathrm{~min}$ before the test). In previous studies from our group, this L-dopa dosage has been found to be effective in producing a significant improvement of motor symptoms in patients with PD (Costa et al., 2003; Koch et al., 2008). The order of the treatment conditions was randomized across PD subjects, that is, half of them performed the experimental task first in the "off" and subsequently in the "on" therapy condition, and half performed the task in reverse order of treatment conditions. The group of normal subjects performed the task only once, without any drug administration. To evaluate the effectiveness of L-dopa administration in ameliorating motor symptoms, PD subjects were given the Unified Parkinson's Disease Rating Scale-section III (UPDRS) (Fahn et al., 1987) immediately before administration of the experimental task in both "off" and "on" therapy conditions. Finally, to investigate possible mood fluctuations as a function of the test-retest evaluation, the Beck Depression Inventory (BDI) (Beck \& Steer, 1987), a self-administered inventory that has shown good reliability in evaluating severity of depression in PD (Visser et al., 2006), was also administered in both "off" and "on" therapy conditions.

\section{Experimental task}

\section{Material}

The experimental material consisted of six triplets of actions the subjects had to perform at the expiration of the established time. In each triplet, the actions were functionally unrelated (see Appendix for a list of the action triplets). The triplets were randomly assigned to the experimental conditions for each individual in the PD and CS groups. PD patients completed three triplets in session 1 and the other three triplets in session 2, while CS completed only three triplets. The examiner and the experimental subject were seated on opposite sides of a table on which the objects the subject had to use to perform the required actions were located. A wall clock was placed to the right of the subject so that he had to turn his head to check it.

\section{Procedure}

The experimental session, which lasted approximately $30 \mathrm{~min}$, consisted of three consecutive trials. At the beginning of each trial, the examiner instructed the participant to perform three actions after $10 \mathrm{~min}$ had elapsed. Participants were informed that they would then have up to $2 \mathrm{~min}$ to

Table 1. Demographic and clinical characteristics of individuals in the PD and CS groups ${ }^{\text {a }}$

\begin{tabular}{|c|c|c|c|c|c|}
\hline \multirow[t]{2}{*}{ Demographic and clinical variables } & \multirow{2}{*}{\multicolumn{2}{|c|}{$\begin{array}{c}\text { PD subjects } \\
N=20 \\
13 / 7\end{array}$}} & \multirow{2}{*}{\multicolumn{2}{|c|}{$\begin{array}{c}\mathrm{CS} \\
N=15 \\
9 / 6\end{array}$}} & \multirow[t]{3}{*}{$\begin{array}{c}F \\
(1,33)\end{array}$} \\
\hline & & & & & \\
\hline $\mathrm{M} / \mathrm{F}$ & Mean & $S D$ & Mean & $S D$ & \\
\hline Age (yrs) & 60.3 & 9.5 & 61.1 & 7.0 & 0.08 \\
\hline Formal education (yr) & 10.0 & 3.2 & 9.7 & 3.6 & 0.07 \\
\hline Age at disease onset $(\mathrm{yr})$ & 54.2 & 11.4 & & & \\
\hline UPDRS ("off" therapy condition) & 24.5 & 9.2 & & & \\
\hline Disease duration (yr) & 6.1 & 6.1 & & & \\
\hline Mini-Mental State Examination & 28.3 & 1.5 & 28.8 & 1.3 & 1.02 \\
\hline Beck Depression Inventory ("off" therapy condition) & 8.8 & 6.3 & 9.7 & 8.6 & 0.14 \\
\hline
\end{tabular}

Note. UPDRS $=$ Unified Parkinson's Disease Rating Scale.

aResults of analyses of variance are also reported. 
initiate the actions (a "forgiveness period"). To be sure the subjects understood what they were expected to do, they had to repeat the examiner's instructions aloud. The examiner made no reference to the possible advantage of checking the clock. During the delay intervals in the three consecutive trials, the subject was engaged in cognitive tasks evaluating selective attention, that is, the Stroop test (Stroop, 1935) and the Attentive Matrices (Spinnler \& Tognoni, 1987); set shifting abilities, that is, the Trail Making test Part A and B (Reitan, 1958); and short-term memory, that is, the Digit span (Orsini et al., 1987). Even if the examiner was giving instructions for the next intervening task at the 10-min time point, the experimental subject always had sufficient time to initiate performance on the prospective task before the 2-min forgiveness period had elapsed. Never did the subject have to interrupt the examiner to initiate task performance. If the subject started to perform the required actions within this time limit, the examiner recorded the actions actually carried out, regardless of whether or not they followed the order indicated by the examiner. If the subject still did not engage in any action by the expiration of the 2-min forgiveness period, the examiner reminded him: "Do you remember that at this point you were supposed to do something?" In the case of an affirmative response, the examiner recorded the number of actions carried out correctly. The same procedure was repeated three times. Each time a different triplet of actions had to be performed. Two separate scores were computed: one for recalling the intention to perform the actions and the other for correctly executing the actions. For intentional recall, a score of 1 was given for each activated intention, and a score of 0 if the intention was lacking (score range of $0-3$ for each experimental session). For action performance, whether or not the intention was recalled or had to be cued, a score of 1 was assigned to any action performed correctly, a score of 0.5 to a partially correct action, and a score of 0 to a completely incorrect or lacking action (score range 0-9). Finally, to investigate subject time-monitoring strategy during the delay interval, the number of clock checks made during each of the five, 2-min subperiods forming the overall 10-min retention interval was also recorded.

\section{RESULTS}

\section{Prospective Memory}

\section{Effect of L-dopa treatment on PD patients' performance}

Preliminary analyses documented that the distribution of the difference scores between the "off" and the "on" treatment conditions in the PD group did not significantly depart from normality (Kolmogorov-Smirnov test: $p>.10$ for both intention retrieval and actions recall). Therefore, the effect of L-dopa treatment on PD patients' performance on the prospective memory task was analyzed by means of a twoway mixed ANOVA with treatment condition ("off" vs. "on") as the within subject factor and order of treatments (on-off vs. off-on) as the between-subject factor.

As regards accuracy of intention retrieval (Figure 1), the performance of PD patients was significantly lower in the "off" than in the "on" therapy condition $(\mathrm{M}=1.7, S D=$ 1.08 ; and $\mathrm{M}=2.4, S D=0.82$, respectively; mean difference between "off" and "on" therapy conditions $=0.75$,

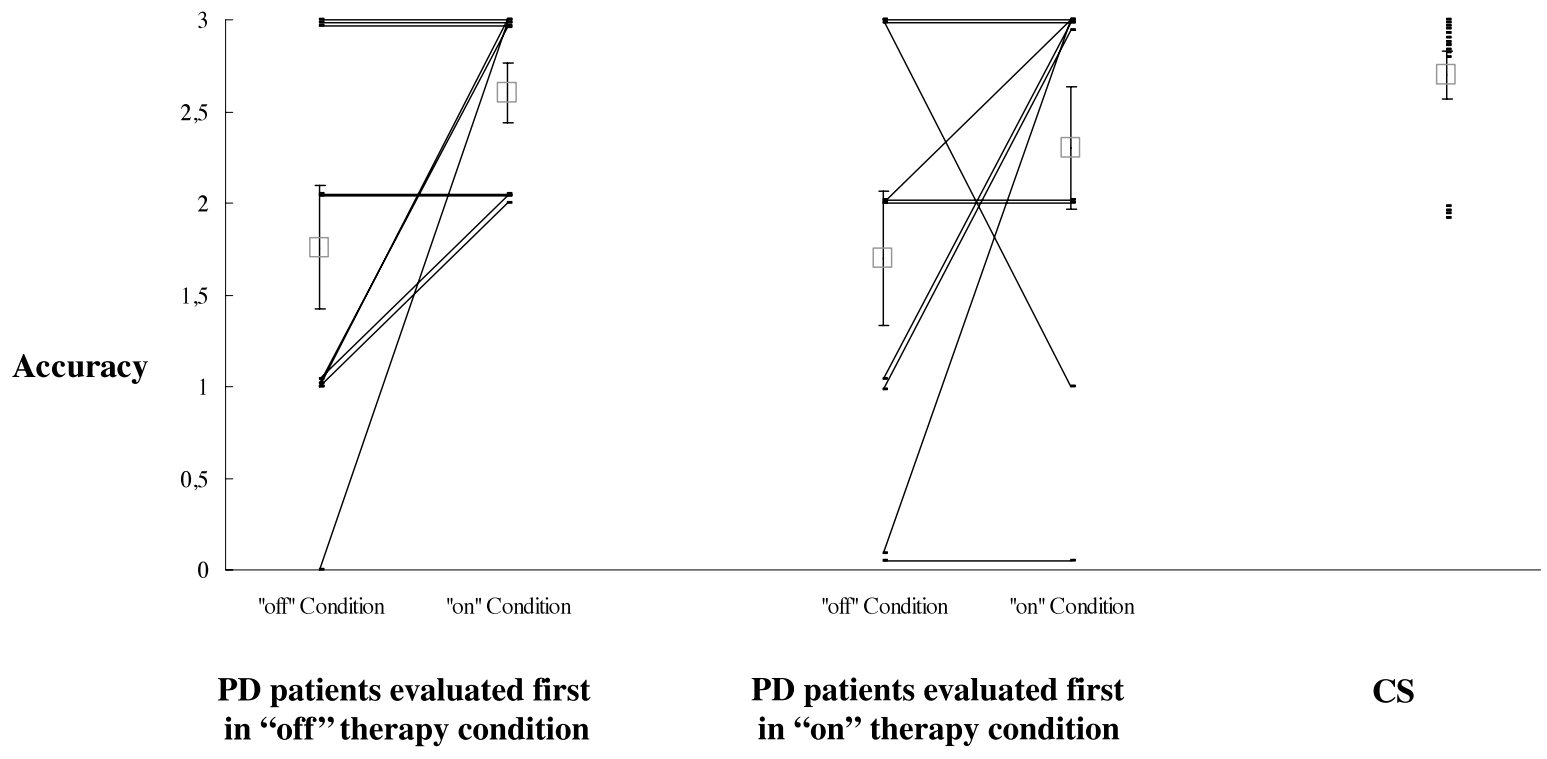

Fig. 1. Performances of the participants in the CS and PD groups on the retrieval of the intention to perform the actions. For PD patients, performances in the "off" therapy (i.e., at least 12 hours after dopamine therapy withdrawal) and in the "on" therapy (i.e., 20-30 min after oral administration of $200 \mathrm{mg}$ of L-dopa) conditions are reported as a function of the order of the treatment condition. Points represent individual subjects. Squares represent the group means while vertical bars represent standard errors. 
$S D=1.25 ; F(1,18)=6.91 ; p=.017$; Cohen's $d=0.78)$. The performance difference between the two conditions was independent of the order of L-dopa treatment, as shown by the lack of significance of both the order of treatment effect $(F(1,18)=0.20 ; p>.60$; Cohen's $d=0.16)$ and the treatment condition by order of treatment interaction $(F(1,18)=$ $0.27 ; p>.60)$. Further confirming the independence of the prospective memory improvement from the order of treatment administration, the number of PD patients whose performance was better in the "on" than in the "off" therapy condition was approximately the same for PD patients who were evaluated first in the "off" condition $(n=5)$ as for PD patients who were evaluated first in the "on" condition $(n=$ 4) (see Figure 1).

As for the recall of the specific actions to be performed (Figure 2), PD subjects performed no better in the "on" condition than in the "off" condition $(\mathrm{M}=8.1, S D=1.11$ and $\mathrm{M}=8.27, S D=1.02$, respectively; mean difference between "off" and "on" therapy conditions $=0.17, S D=$ 1.27). Indeed, neither the main effects of the order of treatment $(F(1,18)=0.50 ; p>.40$; Cohen's $d=0.26)$, treatment condition $(F(1,18)=0.37 ; p>.50$; Cohen's $d=$ 0.16 ), or the order of treatment by treatment condition interaction $(F(1,18)=0.61 ; p>.40)$ approached statistical significance.

\section{PD patients in the "on" therapy condition vs. healthy controls}

The performances of PD subjects in the "on" condition and CS group participants on the prospective memory task were compared by means of two one-way ANOVAs. The group effect was not significant for either the accuracy in intention retrieval $(F(1,33)=1.43 ; p>.20)$ or recall of the specific actions to be performed $(F(1,33)=2.24 ; p>.10)$, thus documenting comparable performances between the two groups.

\section{Additional Analyses}

\section{Effect of L-dopa treatment on time monitoring, performance on intercurrent tasks, and BDI and UPDRS scores of PD patients}

Figure 3 depicts the average number of clock checks made by PD patients in the "off" and "on" treatment conditions during each of the 2-min subperiods of the delay interval in the prospective memory task. To evaluate the effect L-dopa treatment on the frequency of time monitoring in PD patients, a repeated measures ANOVA was executed with treatment condition and time subintervals as within factors. The time subintervals factor was significant $(F(1,76)=2,69 ; p=$ .037). A post hoc Fisher's Least Significant Difference test revealed significant differences in the number of clock checks between the second and the fourth $(p=.02)$ and the fifth $(p<.01)$ sub-periods and between the third and fifth subperiods ( $p=.04$ ). However, neither the treatment condition effect nor the treatment condition by time subintervals interaction approached statistical significance $(F(1,19)=0.53$ and $F(1,76)=0.74$, respectively; $p>.40$ in both cases), thus indicating a lack of difference between the "off" and the "on" therapy conditions in both the overall frequency of clock checks and the strategic varying of clock check frequency as the expiration of the delay interval approached.

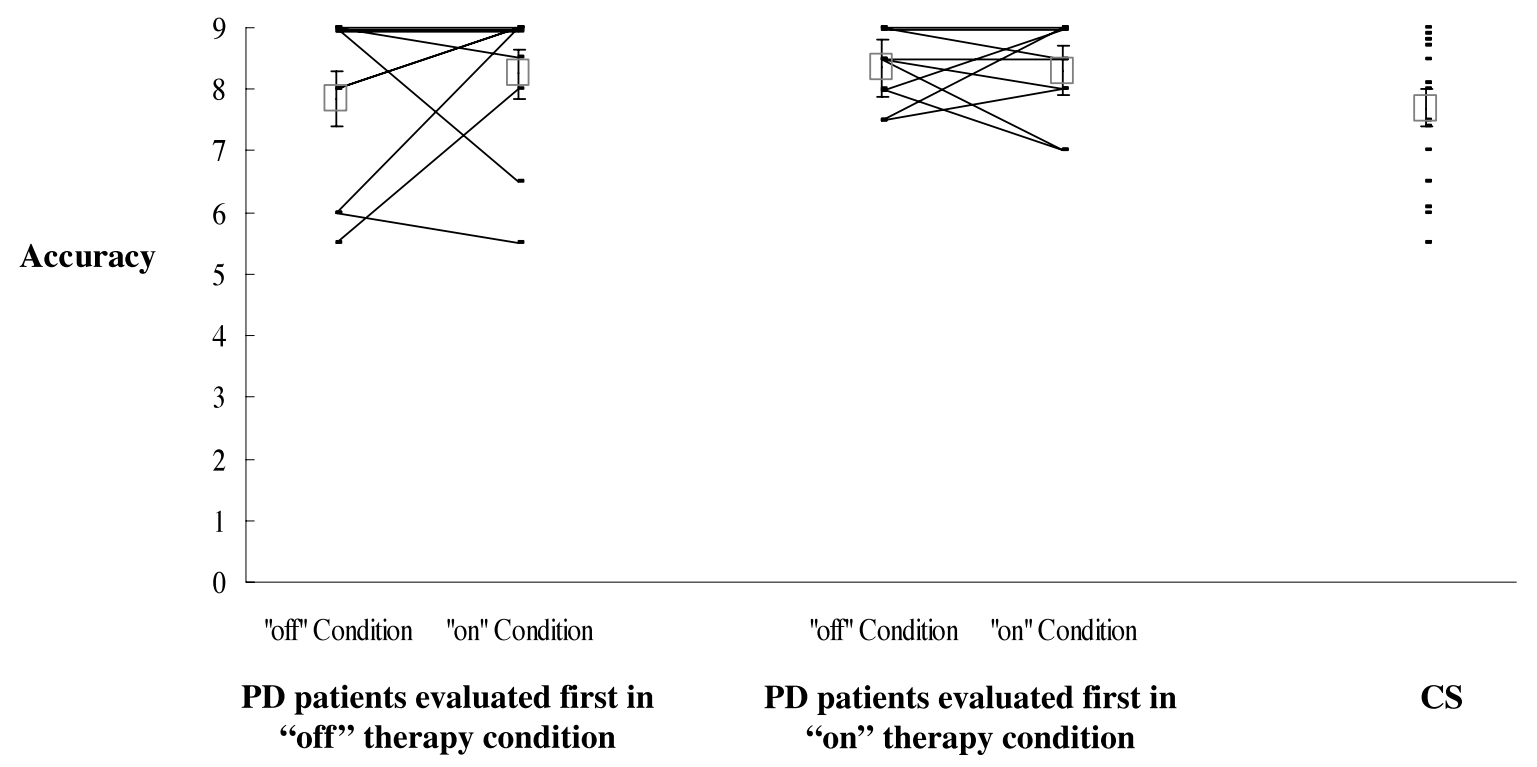

Fig. 2. Performances of the participants in the CS and PD groups on the recall of the specific actions to be performed. PD patients' performances in the "off" therapy (i.e., at least 12 hours after dopamine therapy withdrawal) and in the "on" therapy (i.e., 20-30 min after oral administration of $200 \mathrm{mg}$ of L-dopa) conditions are reported as a function of the order of the treatment conditions. Points represent individual subjects. Squares represent the group means while vertical bars represent standard errors. 


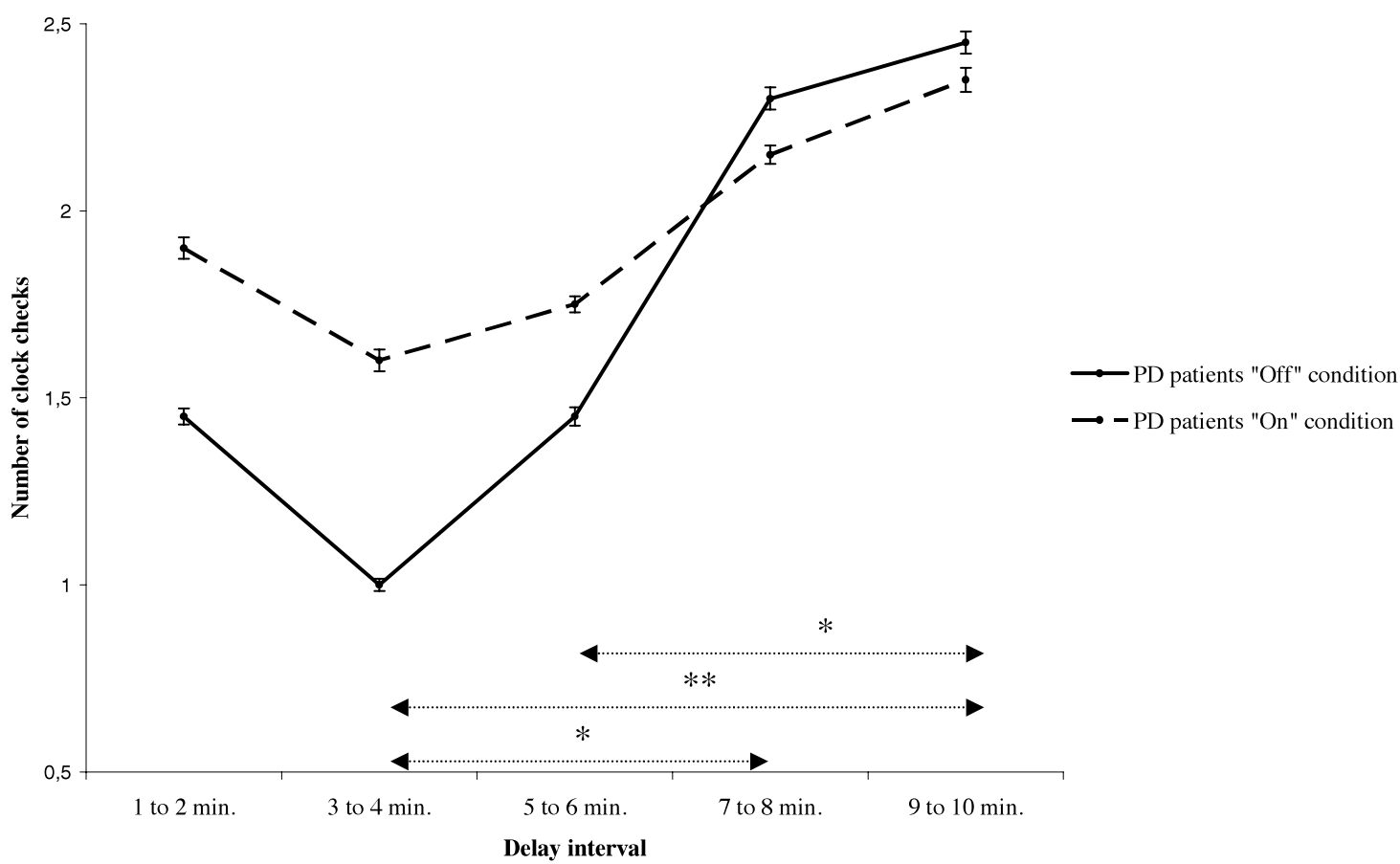

Fig. 3. Average number of clock checks in the individuals with PD (in both "off" and "on" L-dopa treatment conditions) during the delay intervals of the prospective memory task. The overall 10-min period has been fractionated into five, 2-min subperiods. Bars indicate standard errors. Single asterisks indicates statistical significance with $p<.05$; Double asterisks indicates statistical significance with $p<.01$.

A Student $t$ statistic was used to evaluate the effect of L-dopa treatment on PD subjects' scores on the intercurrent tasks. However, due to the large number of measures considered $(N=12)$, the risk of alpha inflation was high in this case. Therefore, Bonferroni correction was applied to these analyses and the $p$ level was set at .004 (i.e., $p=.05 / 12$ ). There were no significant differences in the scores on the intercurrent tasks between the "off" and the "on" therapy conditions (all $p>.02$ ). However, to evaluate whether the PD patients' improvement in intention retrieval observed after L-dopa administration was associated with a performance accuracy change in tasks tapping prefrontal function, we performed a correlation analyses of within-subject difference in intention retrieval ("on" vs. "off" therapy conditions) with within-subject difference in the number of errors committed in the Stroop test-Color-Word condition-, and in the Digit Span Backward and Attentive Matrices scores ("on" vs. "off" therapy conditions). A significant inverse correlation was found with the number of errors in the Stroop test (Pearson's $r=-0.49 ; p=.025$ ), documenting that improved retrieval of the intention to perform the action after L-dopa intake corresponded to a lower number of errors committed on the Stroop test (Figure 4). No other significant correlations were found.

A student $t$ statistic was used to evaluate the effect of L-dopa treatment on PD subjects' score on UPDRS and BDI. The UPDRS score was significantly lower in the "off" than in the "on" therapy condition $(\mathrm{M}=25.5, S D=9.2$ and $\mathrm{M}=12.1, S D=4.5$, respectively; $t(1,19)=9.2 ; p<.001$;
Cohen's $d=1.71$ ). No difference between the "off" and "on" therapy conditions was found on the BDI score $(\mathrm{M}=$ $8.8, S D=6.3$ and $\mathrm{M}=9.6, S D=6.9$, respectively; $t(1,19)=$ $-0.85 ; p>.40$; Cohen's $d=0.11$ ).

\section{Correlations between prospective memory and UPDRS scores in PD patients "on" therapy condition}

A significant inverse correlation was found between the UPDRS score and the number of intentions retrieved on the prospective memory task (Pearson's $r=-0.58 ; p=.007$ ). The number of actions accurately recalled, however, did not correlate with UPDRS scores $(r=0.16)$.

\section{DISCUSSION}

The main aim of the present study was to evaluate the effect of L-dopa treatment on the performance of PD patients on a time-based prospective memory task. Consistent with our hypotheses, we found that the acute administration of $200 \mathrm{mg}$ of L-dopa significantly improved PD participants' accuracy in retrieving the prospective intention and actually normalized their performance. By contrast, our results showed no evidence of an effect of L-dopa on the ability to recall the specific actions to be performed.

Before discussing the possible mechanisms underlying the ameliorative effect of L-dopa treatment on prospective memory functioning in $\mathrm{PD}$, it is worth mentioning a modest 


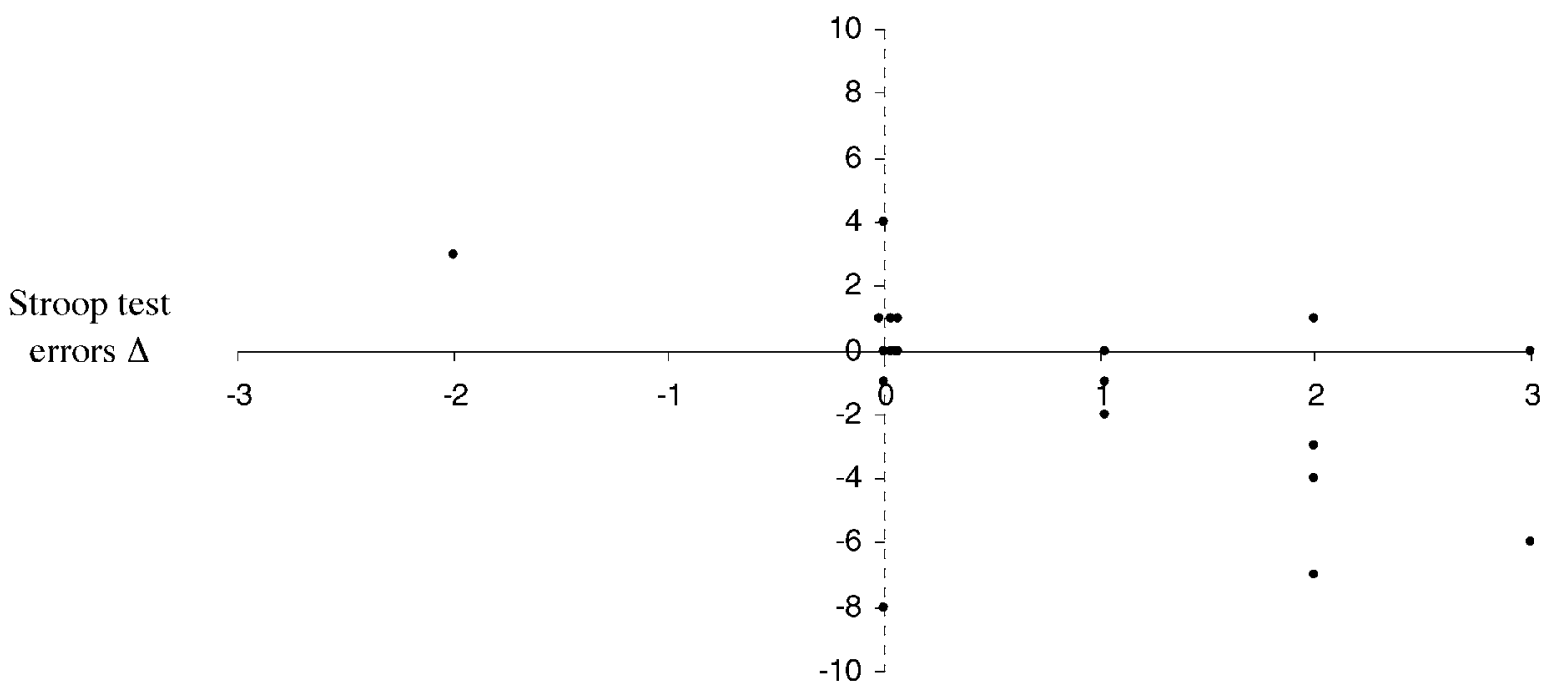

\section{Intention retrieval $\Delta$}

Fig. 4. Relationship between the within subject difference in the number of errors committed in the Stroop Color-Word and the within-subject difference in the intention retrieval in the prospective memory task in the "on" versus "off" therapy condition.

discrepancy between the findings we report here and those in the literature. In contrast to our observation of a complete recovery of prospective memory impairment after L-dopa administration, Katai et al. (2003) and Costa et al. (in press) found a prospective memory deficit in PD patients taking their usual dopaminergic medications. Differences in the way L-dopa was administered to PD patients in the present study and in previously reported studies may explain this discrepancy. First, the time interval between taking L-dopa and performing the experiment was strictly monitored in the present study; it ranged from 20 to $30 \mathrm{~min}$. In previous studies, which did not directly investigate the role of dopaminergic modulation on prospective memory abilities, the time that elapsed between L-dopa administration and prospective memory testing was much longer (approximately $90 \mathrm{~min}$ in Costa et al., in press) or it was not reported at all (Katai et al., 2003).

Based on the previously reported association between prospective memory impairment and cognitive deficits associated with frontal systems alteration, that is, working memory abilities and executive functioning in PD patients (Kliegel et al., 2005; Costa et al., in press), it could be hypothesized that the ameliorative effect of L-dopa on prospective memory functioning was associated with an improvement of such cognitive abilities. Partial support for this hypothesis is provided by the significant correlation between the decrease in the errors committed in the interference condition of the Stroop test and the intention retrieval improvement after L-dopa administration. One possible explanation for this finding is that mechanisms of selective attention played a role in ameliorating PD patients' performance on the prospective memory task. However, performance on tests measuring selective attention and set- shifting abilities did not significantly change as a function of L-dopa treatment. Moreover, PD subjects did not modify their pattern of clock checks as a function of L-dopa treatment, thus suggesting that the ameliorative effect of L-dopa intake on prospective memory was not related to more strategic time monitoring behavior or to an improvement of the repeatedly documented impairment in PD patients' ability to estimate time intervals correctly (Koch et al., 2004, 2008). A second possible explanation derives from the fact that the Stroop color-word interference test pits a volitional intention (to read the color of the ink) against a reactive intention (to read the word). With frontal lesions, the capacity to act on the volitional intention is impaired and patients tend to produce responses based on the reactive intention, which constitute utilization behavior. Thus, failure on both the Stroop color word interference test and on the intention component of our prospective memory test might be attributed to impaired ability to sustain volitional intention, which our study suggests can be corrected through L-dopa administration.

Our data on the beneficial effect of L-dopa on the ability to retrieve the intention to perform planned actions might be explained on the basis of the results of previous work showing that individuals with PD without dementia have specific difficulty in self-initiated strategy recall and task preparation but are not impaired when explicit external cues are provided (Dujardin \& Laurent, 2003; Werheid et al., 2007). In agreement with this view, in the present study L-dopa withdrawal did not affect PD patients' accuracy in the recall of the specific actions to be performed (which, in the absence of self-activation, was prompted by the examiner). Moreover, in a previous study (Costa et al., in press), we found that a group of PD patients was impaired on a 
time-based task, in which the prospective intention retrieval was completely self-initiated, but not in an event-based task, in which the intention retrieval was triggered by an external event. A parallel could be drawn between the deficit in self initiating the prospective intention and the phenomenon of "akinesia paradoxica" held to be characteristic of PD (Jahanshahi et al., 1995; Majsak et al., 1998; Siegert et al., 2002; but see Ballanger et al., 2006, for partially divergent view). This term refers to the fact that PD patients can often initiate movements more quickly in response to external stimuli than voluntarily. It occurs, for example, when a patient with symptoms of akinesia and bradykinesia responds to an alarm by walking rapidly away from the scene. Several studies support these clinical observations, documenting that the ability of patients with PD to begin a motor sequence is more severely impaired (in terms of response times) when they have to rely on self-determined mechanisms than when the drive to act is triggered by an external cue (Jahanshahi et al., 1995; Majsak et al., 1998; Siegert et al., 2002). Overall, these findings are fairly consistent with our finding of a selective impairment after L-dopa withdrawal in the component of the task that specifically requires the subject to self-activate the intention to perform the actions. In substantial agreement with this view, Ridley et al. (2006) recently suggested that PD patients' difficulty in accessing stored information may be a consequence of "a form of intentional neglect, a defect of volition" leading these patients to "fail in self-initiating retrieval strategies although they do not exhibit retrograde amnesia under test conditions which request and thus provoke retrieval." They speculated that such a deficit might be related to dopaminergic loss affecting frontostriatal systems. Indeed, according to Jahanshahi and Frith (1998), intentional self-generated aspects of behavior are mediated by a neural circuitry involving the dorsolateral prefrontal cortex, anterior cingulate, the supplementary motor area, the striatum, and the thalamus.

Our results on a specific association between pharmacological treatment and the prospective memory deficit in PD may be clinically relevant. Subjects whose prospective memory is impaired may have difficulty accomplishing several daily living activities such as taking medicines regularly, keeping appointments or, more generally, taking their chance to act (Burgess et al., 2000; Einstein et al., 1992). In this vein, prospective memory testing in persons with PD could provide relevant information about practical consequences of reduced memory abilities for both the affected individual and his family. Moreover, considering that prospective memory deficits have also been implicated as predictor of the onset of dementia (Huppert \& Beardsall, 1993), longitudinal studies could be useful to evaluate the possible association between prospective memory alterations and the development of dementia in persons with PD.

In conclusion, we observed an improvement of prospective memory abilities in subjects with PD following L-dopa intake. The improvement was not a consequence of better remembering of the actions to be executed and was not related to the ability to implement attentional as well as executive processes; indeed, it was likely the expression of an improved capacity to generate volitional responses. Such an ameliorative effect of L-dopa intake on prospective memory functioning in PD needs to be confirmed in future research. It would be useful, for example, to assess the effect of L-dopa on prospective memory in a dose-response study by varying the interval at which the individual actions are to be retrieved. We cannot say from our data whether the effects mediated by dopamine were the result of cortical or subcortical deficiency. Indeed, this could be a challenging issue to investigate in future studies of PD patients using functional neuroimaging techniques.

\section{ACKNOWLEDGMENTS}

With regard to the present manuscript, no financial or other relationships that could be interpreted as a conflict of interest are present. Moreover, no financial interests or other relationships that include such things as grants or research support, or serving as an employee, consultant, major stock holder or member of a speakers bureau of a corporation are present. Finally, the manuscript has never been published either electronically or in print. Preliminary data from the present research were presented at the 2nd Meeting of the European Societies of Neuropsychology held in Toulouse, France in 2006.

\section{REFERENCES}

Agid, Y., Ruberg, M., Hirsch, E., Raisman-Vozari, R., Vyas, S., Faucheux, B., Michel, P., Kastner, A., Blanchard, V., Damier, P., Villares, J., \& Zhang, P. (1993). Are dopaminergic neurons selectively vulnerable to Parkinson's disease? Advances in Neurology, 60, 148-164.

American Psychiatric Association. (2000). Diagnostic and statistical manual of mental disorders (4th ed. text revision). Washington, DC: American Psychiatric Association Press.

Ballanger, B., Thobois, S., Baraduc, P., Turner, R.S., Broussolle, E., \& Desmurget, M. (2006). "Paradoxical kinesis" is not a hallmark of Parkinson's disease but a general property of the motor system. Movement Disorders, 21, 1490-1495.

Beck, A.T. \& Steer, R.A. (1987). Beck depression inventory manual. San Antonio, TX: Psychological Corporation Press.

Bohnen, N.I., Kaufer, D.I., Hendrickson, R., Ivanco, L.S., Lopresti, B.J., Constantine, G.M., Mathis, Ch.A., Davis, J.G., Moore, R.Y., \& Dekosky, S.T. (2006). Cognitive correlates of cortical cholinergic denervation in Parkinson's disease and parkinsonian dementia. Journal of Neurology, 253, 242-247.

Burgess, P.W. (2000). Strategy application disorder: The role of frontal lobes in human multitasking. Psychological Research, 63, 279-288.

Burgess, P.W., Quayle, A., \& Frith, C.D. (2001). Brain regions involved in prospective memory as determined by positron emission tomography. Neuropsychologia, 39, 545-555.

Burgess, P.W., Scott, S.K., \& Frith, C.D. (2003). The role of the rostral frontal cortex (area 10) in prospective memory: A lateral versus medial dissociation. Neuropsychologia, 41, 906-918.

Burgess, P.W. \& Shallice, T. (1997). The relationship between prospective and retrospective memory: Neuropsychological evidence. In M.A. Conway (Ed.), Cognitive models of memory (pp. 247-272). Hove, UK: Psychology Press. 
Burgess, P.W., Veitch, E., Costello, A., \& Shallice, T. (2000). The cognitive and neuroanatomical correlates of multitasking. Neuropsychologia, 38, 848-863.

Carlesimo, G.A., Casadio, P., \& Caltagirone, C. (2004). Prospective and retrospective components in the memory for actions to be performed in patients with severe closed-head injury. Journal of the International Neuropsychological Society, 10, 679-688.

Cools, R., Stefanova, E., Barker, R.A., Robbins, T.W., \& Owen, A.M. (2002). Dopaminergic modulation of high-level cognition in Parkinson's disease: The role of prefrontal cortex revealed by PET. Brain, 125, 584-594.

Costa, A., Peppe, A., Caltagirone, C., \& Carlesimo, G.A. (in press). Prospective memory impairment in individuals with Parkinson's disease. Neuropsychology.

Costa, A., Peppe, A., Dell'Agnello, G., Carlesimo, G.A., Murri, L., Bonuccelli, U., \& Caltagirone, C. (2003). Dopaminergic modulation of visual-spatial working memory in Parkinson's disease. Dementia and Geriatric Cognitive Disorders, 15, 55-66.

Dujardin, K. \& Laurent, B. (2003). Dysfunction of the human memory systems: Role of the dopaminergic transmission. Current Opinion in Neurology, 16(Suppl 2), 11-16.

Einstein, G.O., Holland, L.J., McDaniel, M.A., \& Guynn, M.J. (1992). Age-related deficits in prospective memory: The influence of task complexity. Psychology and Aging, 1, 471-478.

Einstein, G.O. \& McDaniel, M.A. (1990). Normal aging and prospective memory. Journal of Experimental Psychology. Learning, Memory, and Cognition, 16, 717-726.

Einstein, G.O. \& McDaniel, M.A. (1996). Retrieval processes in prospective memory: Theoretical approaches and some new empirical findings. In M. Brandimonte, G.O. Einstein \& M.A. McDaniel (Ed.), Prospective Memory: Theory and applications (pp. 115-141). Mahwah, NJ: Erlbaum.

Emre, M. (2003). Dementia associated with Parkinson's disease. Lancet Neurology, 2(4), 229-237.

Fahn, S., Elton, R.L., \& Members of the UPDRS Committee (1987). Unified Parkinson's Disease Rating Scale. In S. Fahn, P.D. Marsden, D.B. Calne, \& A. Liebarman (Eds.), Recent developments in Parkinson's disease (pp. 153-163). Florham Park, NJ: MacMillan Health Care Information.

Folstein, M.F., Folstein, S.E., \& McHugh, P.R. (1975). Minimental state: A practical method for grading the cognitive state of patients for the clinician. Journal of Psychiatric Research, 12, 189-198.

Gibb, W.R., Luthert, P.J., Janota, I., \& Lantos, P.L. (1989). Cortical Lewy body dementia: Clinical features and classification. Journal of Neurology, Neurosurgery and Psychiatry, 52, 185-192.

Hoehn, M.M. \& Yahr, M.D. (1967). Parkinsonism: Onset, progression, and mortality. Neurology, 17, 427-442.

Hughes, A.J., Daniel, S.E., Killford, L., \& Lees, A.J. (1992). Accuracy of clinical diagnosis of idiopathic Parkinson's disease: A clinico-pathological study of 100 cases. Journal of Neurology, Neurosurgery, and Psychiatry, 55, 181-184.

Huppert, F.A. \& Beardsall, L. (1993). Prospective memory impairment as an early indicator of dementia. Journal of Clinical and Experimental Neuropsychology, 15, 805-821.

Jahanshahi, M. \& Frith, C. (1998). Willed action and its impairments. Cognitive Neuropsychology, 15, 483-533.

Jahanshahi, M., Jenkins, I., Brown, R., Marsden, C., Passingham, R., \& Brooks, D. (1995). Self-initiated versus externally triggered movements. An investigation using measurement of regional cerebral blood flow with PET and movement-related potentials in normal and Parkinson's disease subjects. Brain, 118, 913-933.

Katai, S., Maruyama, T., Hashimoto, T., \& Ikeda, S. (2003). Event based and time based prospective memory in Parkinson's disease. Journal of Neurology, Neurosurgery, and Psychiatry, 74, 704-709.

Kish, S.J., Shannak, K., \& Hornykiewicz, O. (1988). Uneven patterns of dopamine loss in the striatum of patients with idiopathic Parkinson's disease: Pathophysiologic and clinical implications. The New England Journal of Medicine, 318, 876-880

Kliegel, M. \& Martin, M. (2003). Prospective memory research: Why is it relevant? International Journal of Psychology, 38, 193-194.

Kliegel, M., Phillips, L.H., Lemke, U., \& Kopp, U.A. (2005). Planning and realisation of complex intentions in patients with Parkinson's disease. Journal of Neurology, Neurosurgery, and Psychiatry, 76, 1501-1505.

Knight, R.G. (1998). Prospective memory in aging and neurodegenerative disease. In A.L. Tröster (Ed.), Memory in neurodegenerative disease: Biological, cognitive and clinical perspectives (pp. 172-183). Cambridge: Cambridge University Press.

Koch, G., Costa, A., Brusa, L., Peppe, A., Gatto, I., Torriero, S., Gerfo, E.L., Salerno, S., Oliveri, M., Carlesimo, G.A., \& Caltagirone, C. (2008). Impaired reproduction of second but not millisecond time intervals in Parkinson's disease. Neuropsychologia, [epub ahead of print].

Koch, G., Oliveri, M., Brusa, L., Stanzione, P., Torriero, S., \& Caltagirone, C. (2004). High-frequency rTMS improves time perception in Parkinson disease. Neurology, 63, 2405-2406.

Lewis, S.J., Slabosz, A., Robbins, T.W., Barker, R.A., \& Owen, A.M. (2005). Dopaminergic basis for deficits in working memory but not attentional set-shifting in Parkinson's disease. Neuropsychologia, 43, 823-832.

Majsak, M.J., Kaminski, T., Gentile, A.M., \& Flanagam, J.R. (1998). The reaching movements of patients with Parkinson's disease under self-determined maximal speed and visually cued conditions. Brain, 121, 755-766.

Orsini, A., Grossi, D., Capitani, E., Laiacona, M., Papagno, C., \& Vallar, G. (1987). Verbal and spatial immediate memory span: Normative data from 1355 adults and 1112 children. Italian Journal of Neurological Sciences, 8, 539-548.

Owen, A.M. (2004). Cognitive dysfunction in Parkinson's disease: The role of frontostriatal circuitry. The Neuroscientist, 10, 527-537.

Reitan, R. (1958). Validity of the Trail-Making Test as an indicator of organic brain disease. Perceptual and Motor Skills, 8, 271-276.

Ridley, R.M., Cummings, R.M., Leow-Dyke, A., \& Baker, H.F. (2006). Neglect of memory after dopaminergic lesions in monkeys. Behavioural Brain Research, 166, 253-262.

Siegert, R.J., Harper, D.N., Cameron, F.B., \& Abernethy, D. (2002). Self-initiated versus externally cued reaction times in Parkinson's disease. Journal of Clinical and Experimental Neuropsychology, 24, 146-153.

Spinnler, H. \& Tognoni, G. (1987). Standardizzazione e taratura italiana di test neuropsicologici. Journal of the Neurological Sciences, Suppl. 8.

Stroop, J.R. (1935). Studies of inference in serial verbal reactions. Journal of Experimental Psychology, 18, 643-662. 
Visser, M., Leetjens, A.F., Marinus, J., Stiggelbout, A.M., \& van Hilten, J.J. (2006). Reliability and validity of the Beck depression inventory in patients with Parkinson's disease. Movement Disorders, 21, 668-672.

Werheid, K., Koch, I., Reichert, K., \& Brass, M. (2007). Impaired self-initiated preparation during task switching in Parkinson's disease. Neuropsychologia, 45, 273-281.
Yeterian, E.H. \& Pandia, D.N. (1991). Prefrontostriatal connections in relation to cortical architectonic organisation in rhesus monkey. The Journal of Comparative Neurology, 312, 43-67.

\section{APPENDIX}

Triplets of actions to be performed in the experimental prospective memory task.

1. a) telling the examiner to turn on the computer

b) subject writing his own name on a sheet of paper

c) replacing the telephone receiver

2. a) opening the diary at the current day

b) giving a paper to the examiner

c) reminding the examiner to call the infirmary

3. a) telling the examiner to make a telephone call

b) writing the current date on a piece of paper

c) turning off the light
4. a) telling the examiner to open the door

b) taking a magazine

c) indicating the current date on a calendar

5. a) replacing the pen in the pen box

b) rolling a die

c) throwing a hand cleaner in the waste basket

6. a) replacing a tape cassette in its case

b) taking a pencil

c) attaching a paper to the table using adhesive tape 